\title{
Construction of polynomial solutions to the Dirichlet boundary value problem for the 3-harmonic equation in the unit ball
}

\author{
Valery V. Karachik ${ }^{1, ~ *, ~ S a n j a r ~ A b d o u l a e v ² ~}$ \\ ${ }^{1}$ Department of Mathematical Analysis, South-Ural State University, Chelyabinsk, Russia \\ ${ }^{2}$ Department of Computational Mathematics, South-Ural State University, Chelyabinsk, Russia \\ Email address: \\ karachik@susu.ru (V. V. Karachik); sanjar@mail.ru (S. Abdoulaev)
}

\section{To cite this article:}

Valery V. Karachik, Sanjar Abdoulaev. Construction of Polynomial Solutions to the Dirichlet Boundary Value Problem for the 3-Harmonic Equation in the Unit Ball. Vol. 1, No. 1, 2012, pp. 1-9. doi: 10.11648/j.pamj.20120101.11

\begin{abstract}
Polynomial solution to the Dirichlet boundary value problem for the nonhomogeneous 3-harmonic equation in the unit ball with polynomial right-hand side and polynomial boundary data is constructed. Representation of the Green's function of the Dirichlet boundary value problem in the unit ball in the case of polynomial data is found.
\end{abstract}

Keywords: 3-Harmonic Equation, Almansi Decomposition, Harmonic Polynomials, Dirichlet Boundary Value Problem, Polynomial Solutions

\section{Introduction}

The classical Almansi representation [1] for a polyharmonic function $Q(x)$ has the form

$$
Q(x)=H_{0}(x)+|x|^{2 s} H_{1}(x)+\cdots+|x|^{2 s} H_{s}(x),
$$

where $H_{k}(x)$ are harmonic functions. This representation is successfully used to construct solutions to model boundary value problems for the biharmonic and polyharmonic equations. In numerous studies the Almansi representation is generalized to partial differential operators other than the Laplace operator (see, for example, [2-4]. In this paper, the Almansi representation (1) is first used to construct a solution of the homogeneous Dirichlet boundary value problem for the inhomogeneous 3-harmonic equation $\Delta^{3} u(x)=Q(x)$ (Section 2) and then to construct a solution of the full Dirichlet boundary value problem for the inhomogeneous 3-harmonic equation in the unit ball:

$$
\begin{aligned}
& \Delta^{3} u(x)=Q(x), x \in \Omega ;
\end{aligned}
$$

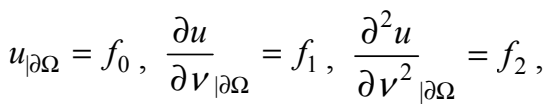

where $\Omega=\left\{x \in \mathbb{R}^{n}:|x|<1\right\}$ and $v$ - is outside normal to $\partial \Omega$ (Section 3$)$.
To construct polynomial solution to a particular Dirichlet boundary value problem for the 3-harmonic equation by the traditional method (see, for example, [5; p.200]) with polynomial boundary data $\left(f_{0}, f_{1}\right.$ and $f_{2}$ - are the traces of polynomials of degree $k$ ) the following scheme is used. Let $G_{j}^{i}(x) \quad, \quad j=1, \ldots, h_{i} \quad, \quad$ where $h_{i}=(1+2 i /(n-2))\left(\begin{array}{c}i+n-3 \\ n-3\end{array}\right)$ (for example, the system from [6]) be a full system of orthonormal on the unit sphere $\partial \Omega \subset \mathbb{R}^{n}$ homogeneous harmonic polynomials of degree $i \leq k$. Taking 3-harmonic polynomials of the form $G_{j}^{i}(x)$, $|x|^{2} G_{j}^{i}(x)$ and $|x|^{4} G_{j}^{i}(x)$ a solution of the Dirichlet boundary value problem is represented in the form

$$
u(x)=\sum_{i=0}^{k} \sum_{j=1}^{h_{i}}\left(C_{j}^{i}+D_{j}^{i}|x|^{2}+E_{j}^{i}|x|^{4}\right) G_{j}^{i}(x) .
$$

Unknown coefficients $C_{j}^{i}, D_{j}^{i}$, and $E_{j}^{i}$ for each $j=1, \ldots, h_{i}$, and $0 \leq i \leq k$ are easily defined from the algebraic system

$$
C_{j}^{i}+D_{j}^{i}+E_{j}^{i}=\int_{\partial \Omega} G_{j}^{i}(s) f_{0}(s) d s,
$$




$$
\begin{gathered}
i C_{j}^{i}+(i+2) D_{j}^{i}+(i+4) E_{j}^{i}=\int_{\partial \Omega} G_{j}^{i}(s) f_{1}(s) d s \\
i(i-1) C_{j}^{i}+(i+2)(i+1) D_{j}^{i}+(i+4)(i+3) E_{j}^{i} \\
=\int_{\partial \Omega} G_{j}^{i}(s) f_{2}(s) d s
\end{gathered}
$$

because a determinant of this system is the Vandermound determinant $W[i, i+2, i+4]$ with factorial powers $t^{[k]}=t(t-1) \cdots(t-k+1) \quad$ [7]. If dimension $n$ and polynomial's degree $k$ are large then this procedure is quite hard work even for the simple polynomials $f_{0}, f_{1}$ and $f_{2}$ because $h_{k} \sim 2 k^{n-2} /(n-2) !, k \rightarrow \infty$ and we have to calculate a lot of surface integrals from (2). In this paper we offer a different method for constructing polynomial solutions to the Dirichlet boundary value problem, which is used a calculation of powers of the Laplace operator on some auxiliary polynomials. Construction of solutions of the Dirichlet boundary value problem in closed form is the prime object of the paper.

In paper [8] with the help of Almansi representation (1) polynomial solutions of the Poisson's equation $\Delta u(x)=Q(x)$ and the polyharmonic equation $\Delta^{m} u(x)=Q(x)$, where $Q(x)$ is an arbitrary polynomial are constructed. On this base in [9] polynomial solution of the Dirichlet boundary value problem and a generalized third boundary value problem for the Poisson's equation are constructed. In [10] the Dirichlet boundary value problem for the biharmonic equation in the unit ball $\Omega$ is considered. In [16] solvability conditions of the Neumann boundary value problem for the biharmonic equation in the unit ball are given and its polynomial solution is constructed. The present paper is a continuation of those investigations for the 3 -harmonic equation $\Delta^{3} u(x)=Q(x)$ in the unit ball $\Omega$. It hopefully allows to solve the Dirichlet problem for the polyharmonic equation in $\Omega$. It is necessary to mention the papers [12-13] devoted to this research area.

In Section 2 of the present paper basing on the properties of the Almansi representation described in Lemmas 1-3, in Lemma 5 the solution of the homogeneous Dirichlet boundary value problem for nonhomogeneous 3-harmonic equation with a simple right-hand side is constructed. Then in Theorem 1 the solution of the same boundary value problem with arbitrary right-hand side is given. In Theorem 2 this solution is simplified. In Theorems 2 and 3 formulas (16) and (18) are derived to simplify calculates of the polynomial solution of the homogeneous Dirichlet boundary value problem for the nonhomogeneous 3-harmonic equation. In Section 3, in Theorem 6, on the base of Theorems 3, 4 and 5 the formula (34) for representation of polynomial solution to the full Dirichlet boundary value problem for the 3-harmonic equation with polynomial data is derived. Solutions to all considered boundary value problems are illustrated by Examples 2-4. To represent the obtained polynomial solutions in the usual form we need to compute powers of the Laplace operator on some special polynomials, defined by the polynomial data of the considered boundary value problem. This procedure can be easily eliminated by applying symbol calculations with the software package "Mathematica" (see Examples 1 and 5).

\section{Polynomial Solution of the Homogeneous Dirichlet Boundary Value Problem for the Inhomogeneous 3-Harmonic Equation}

Consider the following boundary value problem for the inhomogeneous 3-harmonic equation in the unit ball $\Omega=\left\{x \in \mathbb{R}^{n}:|x|<1\right\}$

$$
\begin{aligned}
& \Delta^{3} u(x)=Q(x), \quad x \in \Omega \\
& u_{\mid \partial \Omega}=0, \quad \frac{\partial u}{\partial v \mid \partial \Omega}=0, \quad \frac{\partial^{2} u}{\partial v^{2}}=0
\end{aligned}
$$

with polynomial right-hand side $Q(x)$ and for $n>2$. It was proved in [8, Theorem 3$]$ that some polynomial solution of the 3-harmonic equation (3) can be written in the form

$$
\begin{aligned}
& u(x)=\frac{|x|^{6}}{2 ? ! !} \sum_{k=0}^{\infty} \frac{|x|^{2 k}}{(2 k) ! !(2 k+6) ! !} \int_{0}^{1}(1-\alpha)^{k+2} \\
& \times \alpha^{k+n / 2-1}(-\Delta)^{k} Q(\alpha x) d \alpha .
\end{aligned}
$$

Let us assume that $Q(x)=Q_{m}(x)$ be a homogeneous polynomial of degree $m$. It was shown in [8, Theorem 4] that solution (5) can then be written as

$$
u(x)=\sum_{s=0}^{\infty}(-1)^{s} \frac{(s+2)(s+1)|x|^{2 s+6} \Delta^{s} Q_{m}(x)}{2(2,2)_{s+3}(2 m-2 s+n, 2)_{s+3}}
$$

where $(a, b)_{k}=a(a+b) \cdots(a+(k-1) b)$ is the generalized Pochhammer symbol with $(a, b)_{0}=1$. For example, $(2,2)_{k}=(2 k) ! !$. Note that the denominator of the fraction in the sum involves $(2 m-2 s+n, 2)_{s+3}=(2 m-2 s+n) \cdots$ $(2 m+n+4)$, which does not vanish since $2 s \leq m$. In [8, Theorem 1] it was shown that polynomial solution of the Poisson's equation $\Delta v=Q(x)$ has the form

$$
\begin{aligned}
& v(x)=\frac{|x|^{2}}{2} \sum_{k=0}^{\infty} \frac{|x|^{2 k}}{(2 k) ! !(2 k+2) ! !} \int_{0}^{1}(1-\alpha)^{k} \\
& \times \alpha^{k+n / 2-1}(-\Delta)^{k} Q(\alpha x) d \alpha .
\end{aligned}
$$

Besides, it was shown in [8, Theorem 2] that for $Q(x)=Q_{m}(x)$ the solution (7) can be written in the different form 


$$
v(x)=\sum_{s=0}^{\infty}(-1)^{s} \frac{|x|^{2 s+2} \Delta^{s} Q_{m}(x)}{(2,2)_{s+1}(2 m-2 s+n, 2)_{s+1}} .
$$

Consider the following operator

$$
\begin{aligned}
& \Delta_{-m}=\frac{|x|^{2 m}}{2(2 m-2) ! !} \sum_{k=0}^{\infty} \frac{|x|^{2 k}}{(2 k) ! !(2 k+2 m) ! !} \\
& \left.\times \int_{0}^{1}(1-\alpha)^{k+m-1} \alpha^{k+n / 2-1}(-\Delta)^{k} ? \alpha x\right) d \alpha
\end{aligned}
$$

It was proved in [8,Theorem 3] that the polynomial $u(x)=\Delta_{-k} Q(x)$ is a solution to the polyharmonic equation $\Delta^{k} u=Q(x)$, i.e. $\Delta^{k} \Delta_{-k} Q(x)=Q(x)$. Thus the solution (5) can be written in the short form $u(x)=\Delta_{-3} Q(x)$.

Lemma 1. Let $Q_{l}(x)$ be a homogeneous polynomial of degree $l$, then the following equality holds

$$
\begin{gathered}
\left(\Delta_{-1}\right)^{m} Q_{l}(x)=|x|^{2 m} \sum_{k=0}^{\infty}(-1)^{k}\left(\begin{array}{c}
k+m-1 \\
m-1
\end{array}\right) \\
\times \frac{|x|^{2 k} \Delta^{k} Q_{l}(x)}{(2,2)_{k+m}(n+2 l-2 k, 2)_{k+m}}
\end{gathered}
$$

In [10, Lemma 1] it was proved that Lemma 1 holds true for $m=2$ and in [8, Theorem 2] is obtained that it is true for $m=1$ also.

Proof. It is proved in [10, Theorem 3] that $\Delta_{-m}=\Delta_{-(m-1)} \Delta_{-1}$ and therefore by induction $\Delta_{-m}=\left(\Delta_{-1}\right)^{m}$. Besides in [10, Theorem 4] is obtained that

$$
\begin{gathered}
\Delta_{-m} Q_{l}(x)=|x|^{2 m} \sum_{k=0}^{\infty}(-1)^{k}\left(\begin{array}{c}
k+m-1 \\
m-1
\end{array}\right) \\
\times \frac{|x|^{2 k} \Delta^{k} Q_{l}(x)}{(2,2)_{k+m}(n+2 l-2 k, 2)_{k+m}}
\end{gathered}
$$

Therefore

$$
\begin{gathered}
\left(\Delta_{-1}\right)^{m} Q_{l}(x)=\Delta_{-m} Q_{l}(x)=|x|^{2 m} \sum_{k=0}^{\infty}(-1)^{k}\left(\begin{array}{c}
k+m-1 \\
m-1
\end{array}\right) \\
\times \frac{|x|^{2 k} \Delta^{k} Q_{l}(x)}{(2,2)_{k+m}(n+2 l-2 k, 2)_{k+m}}
\end{gathered}
$$

Lemma is proved.

Consider the polyharmonic equation with a particular right-hand side

$$
\left.\Delta^{k} u=|x|^{2 m} ? P_{s} x\right), \quad x \in D,
$$

where $P_{s}(x)$ is a homogeneous harmonic polynomial of degree $s$, and $D \subset \mathbb{R}^{n}$ is a star-shaped domain centered at the origin. From the results of the paper [9, Theorem 3] it follows that solution of the equation $\left.\Delta v=|x|^{2 m} P_{s} x\right)$, written in the form (8) can be also written as

$$
v(x)=\frac{\left.|x|^{2 m+2} ? P_{s} x\right)}{(2 m+2)(2 m+2 s+n)}
$$

or

$$
\left.\Delta_{-1}\left(|x|^{2 m} P_{s} x\right)\right)=\frac{\left.|x|^{2 m+2} P_{s} x\right)}{(2 m+2)(2 m+2 s+n)} .
$$

More general statement is true.

Lemma 2. The following equality holds

$$
\left.\Delta_{-k}\left(|x|^{2 m} P_{s} x\right)\right)=\frac{\left.|x|^{2 m+2 k} P_{s} x\right)}{(2 m+2,2)_{k}(2 m+2 s+n, 2)_{k}} .
$$

Proof. As was mentioned above (see Lemma 1)

$$
\Delta_{-k}=\Delta_{-(k-1)} \Delta_{-1}
$$

and therefore

$$
\begin{gathered}
\left.\left.\Delta_{-k}\left(|x|^{2 m} P_{s} x\right)(=) \Delta_{-1}\right|^{k}(x)^{2 m} P_{f} x ?\right) \\
=\left(\Delta_{-1}\right)^{k-1} \Delta_{-1}\left(|x|^{2 m} P_{s}(x)\right) \\
=\frac{\left(\Delta_{-1}\right)^{k-1}\left(|x|^{2 m+2} P_{s}(x)\right)}{(2 m+2)(2 m+2 s+n)} \\
=\cdots=\frac{|x|^{2 m+2 k} P_{s} x \quad()}{(2 m+2,2)_{k}(2 m+2 s+n, 2)_{k}}
\end{gathered}
$$

Lemma is proved.

Let us decompose a homogeneous polynomial $Q_{m}(x)$ by means of Almansi formula (1) into the terms of the form $|x|^{2 s} R_{m-2 s}(x)$

$$
\begin{aligned}
& Q_{m}(x)=R_{m}(x)+|x|^{2} R_{m-2}(x)+\cdots \\
& +|x|^{2 s} R_{m-2 s}(x), \quad m-2 s \geq 0,
\end{aligned}
$$

where $R_{k}(x)$ are homogeneous harmonic polynomials. If we apply to both sides of this equality the operator $\Delta_{-k}$ then, by Lemma 2, the solution of the equation $\Delta^{k} v(x)=Q_{m}(x)$ given by formula (6) has the form

$$
v(x)=\sum_{s=0}^{[m / 2]} \frac{|x|^{2 s+2 k} R_{m-2 s}(x)}{(2 s+2,2)_{k}(2 m-2 s+n, 2)_{k}},
$$

where $[a]$ is the integer part of $a$ and the homogeneous harmonic polynomials $R_{k}(x)$ are determined by the 
Almansi formula (12). From the explicit form of polynomials $R_{k}(x)$, obtained in [11], similarly to formula (12) the following assertion is true.

Lemma 3 [9] The harmonic polynomials $R_{m-2 k}(x)$ in the Almansi representation (1) of the homogeneous polynomial $Q_{m}(x)$ have the form

$$
\begin{gathered}
R_{m-2 k}(x)=\frac{2 m-4 k+n-2}{(2,2)_{k}} \\
\times \sum_{s=0}^{\infty} \frac{(-1)^{s}|x|^{2 s} \Delta^{s+k} Q_{m}(x)}{(2,2)_{s}(2 m-4 k-2 s+n-2,2)_{s+k+1}} .
\end{gathered}
$$

Let us investigate the Dirichlet boundary value problem (3)-(4) with $Q(x)=|x|^{2 s} R_{m-2 s}(x)$. Consider the operator

$$
\Lambda u(x)=\sum_{k=1}^{n} x_{k} u_{x_{k}}(x)
$$

which is defined on functions from $C^{1}(\Omega)$. It has the following easy to verify properties: if $u$ is a harmonic function in $\Omega$, then function $\Lambda u$ is also a harmonic function in $\Omega$; the equality $\Lambda(u v)=v \Lambda u+u \Lambda v$ holds; if $P_{m}(x)$ is a homogeneous polynomial of degree $m$, then $\Lambda P_{m}(x)=m P_{m}(x)$. We formulate one more important property of the operator $\Lambda$.

Lemma 4. On the unit sphere $\partial \Omega$ the following equality holds

$$
{\frac{\partial^{k} u}{\partial v^{k}}}_{\partial \Omega}=\Lambda^{[k]} u_{\mid \partial \Omega}
$$

where factorial power is defined by the equality $t^{[k]}=t(t-1) \cdots(t-k+1) \quad[7]$.

Let $P_{k}(t)=\sum_{s=0}^{k} c_{s} t^{s}$ be a polynomial. We define a factorial polynomial, associated to the polynomial $P_{k}(t)$ by the equality $P_{[k]}(t)=\sum_{s=0}^{k} c_{s} t^{[s]}[14]$.

Corollary 1 . The following equality holds

$$
P_{k}\left(\frac{\partial}{\partial v}\right) u_{\mid \partial \Omega}=P_{[k]}(\Lambda) u_{\mid \partial \Omega}
$$

To prove this statement we apply Lemma 4 to the each term of the polynomial $P_{k}\left(\frac{\partial}{\partial v}\right) u_{\mid \partial \Omega}$.

It is not hard to prove the following statement.

Lemma 5. The solution $v_{s}(x)$ of the homogeneous Dirichlet boundary value problem (3)-(4) with
$Q(x)=|x|^{2 s} R_{m-2 s}(x)$ has the form

$$
\begin{gathered}
v_{s}(x)=C_{m, s}\left(|x|^{2 s+6}-\frac{(s+1)(s+2)}{2}\right. \\
\left.+(s+1)(s+3)|x|^{2}-\frac{(s+2)(s+3)}{2}|x|^{4}\right) R_{m-2 s}(x)
\end{gathered}
$$

where $1 / C_{m, s}=(2 s+2,2)_{3}(2 m-2 s+n, 2)_{3}$.

We transform the obtained solution $u_{0}(x)$.

Theorem 1. Let $A=m+n / 2$ and

$$
\begin{gathered}
A_{s, \alpha}=(s-\alpha+1)(s-\alpha+2)(A-2 s+2 \alpha-1)(A-2 s+\alpha-3) \\
\times(A-2 s+\alpha-2)+2(s-\alpha+2) \alpha(A-2 s+2 \alpha-3) \\
\times(A-2 s+\alpha-3)(A-2 s+2 \alpha-3)(A-2 s+\alpha-3) \\
\times(A-s+\alpha+2)+\alpha(\alpha-1)(A-2 s+2 \alpha-5) \\
\times(A-s+\alpha+2)(A-s+\alpha+1)
\end{gathered}
$$

then the following equality holds

$$
u_{0}(x)=\sum_{s=0}^{\infty} \frac{\Delta^{s} Q_{m}(x)}{2 ?^{s+3}} \sum_{\alpha=0}^{s+3} \frac{(-1)^{\alpha+1} A_{s, \alpha}|x|^{2 \alpha}}{\alpha !(s-\alpha+3) !(A-2 s+\alpha-3)_{s+6}}
$$

where $(a)_{s}=a(a+1) \cdots(a+s-1)$ is the Pochhammer symbol.

From the obtained formula (15) we cannot see that the polynomial $u_{0}(x)$ obtained from (15) satisfies to the homogeneous conditions (4)

$$
u_{0}(x)_{|x|=1}=0, \frac{\partial u_{0}(x)}{\partial v_{|x|=1}}=0,{\frac{\partial^{2} u_{0}(x)}{\partial v^{2}}}_{|x|=1}=0 .
$$

Theorem 2. The solution $u_{0}(x)$ of the problem (3)-(4) with $Q(x)=Q_{m}(x)$ can be written as

$$
\begin{aligned}
u_{0}(x)= & \left(|x|^{2}-1\right)^{3} \sum_{s=0}^{\infty} \frac{(s+1)(s+2) \Delta^{s} Q_{m}(x)}{2 ?^{s+3} s+} \\
& \times \sum_{k=0}^{s}(-1)^{k}\left(\begin{array}{l}
s \\
k
\end{array}\right) \frac{|x|^{2 k}}{(A-2 s+k)_{s+3}},
\end{aligned}
$$

where we used $A=m+n / 2$ for short as in Theorem 1 .

Proof of this Theorem is similar to proof of Theorem 2 from [10].

Lemma 6. Let the function $v(x)$, defined in $\bar{\Omega}$ be written in the form $v_{0}(x)=\left(|x|^{2}-1\right)^{l} S(x)$, where $S(x) \in C^{l-1}(\bar{\Omega})$ and $l \in \mathbb{N}$. Then it satisfies the condition

$$
P_{l-1}\left(\frac{\partial}{\partial v}\right) v(x)_{\mid \partial \Omega}=0
$$


where $P_{l-1}(t)$ is an arbitrary polynomial of degree $l-1$ and hence $v(x)$ satisfies the homogeneous Dirichlet's conditions on $\partial \Omega$

$$
v_{\mid \partial \Omega}=0, \quad \frac{\partial v}{\partial v \mid \partial \Omega}=0, \ldots,{\frac{\partial^{l-1} v}{\partial v^{l-1} \mid \partial \Omega}}_{|c|}=0
$$

Proof. Indeed because of equality $\Lambda(u v)=v \Lambda u+u \Lambda v$ we can write

$$
\begin{gathered}
\Lambda v(x)=\Lambda\left(|x|^{2}-1\right)^{l} S(x) \\
=l S(x)\left(|x|^{2}-1\right)^{l-1} \Lambda\left(|x|^{2}-1\right)+\left(|x|^{2}-1\right)^{l} \Lambda S(x) \\
=\left(|x|^{2}-1\right)^{l-1}\left(2 l S(x)|x|^{2}\right. \\
\left.+\left(|x|^{2}-1\right) \Lambda S(x)\right) \equiv\left(|x|^{2}-1\right)^{l-1} S_{1}(x)
\end{gathered}
$$

where $S_{1}(x) \in C^{l-2}(\bar{\Omega})$ and therefore $\Lambda v(x)_{\mid \partial \Omega}=0$. If we continue similarly then we obtain $\Lambda^{l-1} v(x)=$ $=\Lambda^{l-1}\left(|x|^{2}-1\right)^{l} S(x)=\left(|x|^{2}-1\right) S_{l-1}(x) \quad, \quad$ where $S_{l-1}(x) \in C(\bar{\Omega})$ and then $\Lambda^{l-1} v(x)_{\mid \partial \Omega}=0$. Therefore if $P_{l-1}(t)$ - is an arbitrary polynomial of degree $l-1$ then by Corollary 1 we have

$$
P_{l-1}\left(\frac{\partial}{\partial v}\right) v(x)_{\mid \partial \Omega}=P_{[l-1]}(\Lambda) v(x)_{\mid \partial \Omega}=0 .
$$

Lemma is proved.

On the base of Lemma 6 the polynomial $u_{0}(x)$ from Theorem 2 satisfies the homogeneous Dirichlet's conditions (4).

Remark 1. Formulas defined solution of the homogeneous Dirichlet problem for the harmonic equation $u_{1}(x)$ [9], biharmonic equation $u_{2}(x)$ [10] and 3 -harmonic equation $u_{3}(x)$ (16) very similar each other and can be written for $l=1,2,3$ in the one form

$$
\begin{gathered}
u_{l}(x)=\left(|x|^{2}-1\right)^{l} \sum_{s=0}^{\infty}\left(\begin{array}{c}
s+l-1 \\
l-1
\end{array}\right) \frac{\Delta^{s} Q_{m}(x)}{4^{s+l}(s+l) !} \\
\times \sum_{k=0}^{s}(-1)^{k}\left(\begin{array}{l}
s \\
k
\end{array}\right) \frac{|x|^{2 k}}{(m-2 s+k+n / 2)_{s+l}} .
\end{gathered}
$$

Example 1. The solution of the Dirichlet problem (3)-(4) with $Q_{6}(x)=x_{1}^{3} x_{2} x_{3}^{2}$, written in the form (16) can be easily calculated with the help of symbolic package "Mathematica" and has the form

$$
u\left(x_{1}, x_{2}, x_{3}\right)=-\frac{x_{1} x_{2}\left(x_{1}^{2}+x_{2}^{2}+x_{3}^{2}-1\right)^{3}}{4655851200}
$$

$$
\begin{gathered}
\times\left(-1292+903 x_{1}^{4}-252 x_{2}^{4}-4921 x_{3}^{2}+3213 x_{3}^{4}+\right. \\
\left.+7 x_{1}^{2}\left(-133+93 x_{2}^{2}-2272 x_{3}^{2}\right)+7 x_{2}^{2}\left(152+423 x_{3}^{2}\right)\right) .
\end{gathered}
$$

We again slightly transform polynomial $u_{0}(x)$ - the solution of the Dirichlet problem (3)-(4) with $Q(x)=Q_{m}(x)$ in such a way that then to have a possibility to obtain a formula for the arbitrary polynomial $Q(x)$.

Lemma 7. It is true that

$$
\begin{gathered}
u_{0}(x)=\frac{\left(|x|^{2}-1\right)^{3}}{16} \\
\times \sum_{s=0}^{\infty} \int_{0}^{1} \frac{\left(1-t|x|^{2}\right)^{s}(1-t)^{s+2}}{(2 s) ! !(2 s+6) ! !} \Delta^{s} Q_{m}(t x) t^{n / 2-1} d t
\end{gathered}
$$

Proof is similar to proof of formula (21) from [9].

Let us solve the Dirichlet problem (3)-(4) with an inhomogeneous polynomial $Q(x)$.

Theorem 2. The solution of the Dirichlet problem (3)-(4) can be written as

$$
\begin{gathered}
u(x)=\frac{\left(|x|^{2}-1\right)^{3}}{16} \\
\times \int_{0}^{1} \sum_{s=0}^{\infty} \frac{\left(1-\alpha|x|^{2}\right)^{s}(1-\alpha)^{s+2}}{(2 s) ! !(2 s+6) ! !} \Delta^{s} Q(\alpha x) \alpha^{n / 2-1} d \alpha
\end{gathered}
$$

Proof. Let $Q(x)$ be an arbitrary polynomial. It can be represented as the sum of homogeneous terms $Q(x)=\sum_{m} Q_{m}(x)$. Denote by $u_{m}(x)$ the polynomial solution of the Dirichlet problem (3)-(4) with the right-hand side $Q(x)=Q_{m}(x)$. Then obviously the desired solution has the form $u(x)=\sum_{m} u_{m}(x)$. It follows from (17) that

$$
\begin{gathered}
u(x)=\sum_{m} u_{m}(x)=\frac{\left(|x|^{2}-1\right)^{3}}{16} \\
\times \sum_{s=0}^{\infty} \int_{0}^{1} \frac{\left(1-\alpha|x|^{2}\right)^{s}(1-\alpha)^{s+2}}{(2 s) ! !(2 s+6) ! !} \alpha^{n / 2-1} \Delta^{s} \sum_{m} Q_{m}(\alpha x) d \alpha \\
=\frac{\left(|x|^{2}-1\right)^{3}}{16} \\
\times \int_{0}^{1} \sum_{s=0}^{\infty} \frac{\left(1-\alpha|x|^{2}\right)^{s}(1-\alpha)^{s+2}}{(2 s) ! !(2 s+6) ! !} \Delta^{s} Q(\alpha x) \alpha^{n / 2-1} d \alpha
\end{gathered}
$$

Theorem is proved.

Remark 2. The Green's function (operator) of the Dirichlet problem (3)-(4) in the unit ball in the case of 
polynomial functions $Q(x)$ can be written as

$$
\begin{gathered}
G[Q](x ; \alpha) ? \frac{\left(|x|^{2}-1\right)^{3}}{16} \\
\times \sum_{s=0}^{\infty} \frac{\left(1-\alpha|x|^{2}\right)^{s}(1-\alpha)^{s+2}}{(2 s) ! !(2 s+6) ! !} \alpha^{n / 2-1}\left(\Delta^{s} Q\right)(\alpha x)
\end{gathered}
$$

and then the solution from (18) has the form

$$
u(x)=\int_{0}^{1} G[Q](x, \alpha) d \alpha
$$

Example 2. Let us check the formulas (18) and (15). Let $Q(x)=x_{i}$ in the Dirichlet problem (3)-(4) and, hence, $m=1$. Then the sum in (18) has the only term indexed by $s=0$. We obtain

$$
\begin{gathered}
u_{0}(x)=\frac{\left(|x|^{2}-1\right)^{3}}{16} \int_{0}^{1} \frac{(1-\alpha)^{2}}{2 ?}\left(\alpha x_{i}\right) \alpha^{n / 2-1} d \alpha \\
=x_{i} \frac{\left(|x|^{2}-1\right)^{3}}{48(n+2)(n+4)(n+6)} .
\end{gathered}
$$

Is this the solution of our problem? According to Lemma 6 the homogeneous conditions (4) are fulfilled. Using equality (see [11])

$$
\begin{gathered}
\Delta\left(|x|^{2 s} P(x)\right)=2 s|x|^{2 s-2} \\
\times(2 \Lambda+2 s+n-2) P(x)+|x|^{2 s} \Delta P(x)
\end{gathered}
$$

with $P(x)=x_{i}, s=3$ and the properties of the operator $\Lambda$ we obtain

$$
\begin{gathered}
\Delta^{3} u_{0}(x)=\Delta^{3} \frac{x_{i}|x|^{6}}{48(n+2)(n+4)(n+6)} \\
=\Delta^{2} \frac{6(n+6) x_{i}|x|^{4}}{48(n+2)(n+4)(n+6)} \\
=\Delta \frac{4(n+4) x_{i}|x|^{2}}{8(n+2)(n+4)}=\frac{2(n+2) x_{i}|x|^{2}}{2(n+2)}=x_{i}
\end{gathered}
$$

Note that formula (18) by its way of representation of the solution is similar to representation of a solution of Cauchy problem for ODEs with constant coefficients obtained in [15].

\section{Polynomial Solution of the}

Inhomogeneous Dirichlet Boundary Value Problem for the Homogeneous 3-Harmonic Equation
Now consider the following Dirichlet problem for the 3 -harmonic equation in the unit ball $\Omega=\left\{x \in \mathbb{R}^{n}:|x|<1\right\}$

$$
\begin{gathered}
\Delta^{3} u(x)=0, \quad x \in \Omega \\
u_{\partial \Omega \Omega}=P(x), \quad \frac{\partial u}{\partial v}_{\partial \Omega}=0, \quad{\frac{\partial^{2} u}{\partial v^{2}}}_{\partial \Omega}=0,
\end{gathered}
$$

where $v$ is outside normal to $\partial \Omega$, with a polynomial boundary value $P(x)$ and for $n>2$.

Together with the polynomial $P(x)$ consider two associated with it polynomials

$$
\begin{array}{r}
P_{(0)}(x)=P(x)+\frac{1-|x|^{2}}{2} \Lambda P(x) \\
+\frac{\left(1-|x|^{2}\right)^{2}}{8}\left(\Lambda^{2}+2 \Lambda\right) P(x), \\
P_{(1)}^{\alpha, s}(x)=\left(\Delta\left(\Lambda^{2}+2 \Lambda\right)-2 \frac{1-\alpha}{2 s+4} \Delta^{2} \Lambda\right. \\
\left.+\frac{(1-\alpha)^{2}}{(2 s+4)(2 s+6)} \Delta^{3}\right) P(x)
\end{array}
$$

where $\alpha \in[0,1]$ and $s \in \mathbb{N}_{0} \equiv \mathbb{N} \cup\{0\}$. The result below complements Theorem 3 .

Theorem 4. The solution of the problem (20)-(21) can be written in the form

$$
v(x)=P_{(0)}(x)+\frac{\left(1-|x|^{2}\right)^{3}}{16}
$$

$$
\times \int_{0}^{1} \sum_{s=0}^{\infty} \frac{\left(1-\alpha|x|^{2}\right)^{s}(1-\alpha)^{s}}{(2 s) ! !(2 s+2) ! !}\left(\Delta^{s} P_{(1)}^{\alpha, s}\right)(\alpha x) \alpha^{n / 2-1} d \alpha
$$

Example 3. Let $P(x)=x_{j}^{2}$ in the problem (20)-(21). Then the sum in (24) has the only term indexed by $s=0$. Since $\Lambda x_{j}^{2}=2 x_{j}^{2}$ and $\Delta x_{j}^{2}=2$, then

$$
\begin{gathered}
P_{(0)}(x)=P(x)+\frac{1-|x|^{2}}{2} \Lambda P(x)+\frac{\left(1-|x|^{2}\right)^{2}}{8}\left(\Lambda^{2}+2 \Lambda\right) P(x) \\
=x_{j}^{2}\left(1+\left(1-|x|^{2}\right)+\left(1-|x|^{2}\right)^{2}\right), \\
P_{(1)}^{\alpha, 0}(x)=\left(\Delta\left(\Lambda^{2}+2 \Lambda\right)-2 \frac{1-\alpha}{4} \Delta^{2} \Lambda+\frac{(1-\alpha)^{2}}{4 ?} \Delta_{6}^{3}\right) P(x) \\
=16 .
\end{gathered}
$$

By formula (24) we find 


$$
\begin{gathered}
v(x)=x_{j}^{2}\left(3-3|x|^{2}+|x|^{4}\right)+\frac{\left(1-|x|^{2}\right)^{3}}{16} \int_{0}^{1} \frac{1}{2} ? 6 \alpha^{n / 2-1} d \alpha \\
=x_{j}^{2}\left(3-3|x|^{2}+|x|^{4}\right)++\frac{1}{n}\left(1-|x|^{2}\right)^{3} \\
=3 x_{j}^{2}-3 x_{j}^{2}|x|^{2}+x_{j}^{2}|x|^{4} \\
-\frac{1}{n}\left(|x|^{6}-3|x|^{4}+3|x|^{2}-1\right)
\end{gathered}
$$

Let us check that the obtained polynomial $v(x)$ is really the solution of the problem (20)-(21) with $P(x)=x_{j}^{2}$. Using (19) we obtain

$$
\begin{aligned}
\Delta v(x)= & 6-6(n+4) x_{j}^{2}-6|x|^{2}+4(n+6) x_{j}^{2}|x|^{2}+2|x|^{4} \\
& -\left(6(n+4)|x|^{4}-12(n+2)|x|^{2}+6 n\right) / n
\end{aligned}
$$

Then

$$
\begin{aligned}
& \Delta^{2} v(x)=-12(n+4)-12 n+8(n+6)(n+4) x_{j}^{2} \\
& +8(n+6)|x|^{2}+8(n+2)|x|^{2} \\
& -\left(24(n+4)(n+2)|x|^{2}-24(n+2) n\right) / n
\end{aligned}
$$

and therefore polynomial $v(x)$ is a 3-harmonic polynomial:

$$
\begin{gathered}
\Delta^{3} v(x)=16(n+4)(n+6)+ \\
16 n(n+2)+16 n(n+6)-\frac{48 n(n+2)(n+4)}{n} \\
=16(n+4) \times(n+6)+32 n(n+4)-48(n+2)(n+4) \\
=16(n+4)(n+6+2 n-3 n-6)=0
\end{gathered}
$$

In addition, from (25) it follows that $v(x)$ satisfies the conditions $v_{|x|=1}=\left(x_{j}^{2}\right)_{|x|=1}$,

$$
\begin{gathered}
\frac{\partial v}{\partial v_{|x|=1}}=\Lambda v_{|x|=1}= \\
6\left(x_{j}^{2}-2 x_{j}^{2}|x|^{2}+x_{j}^{2}|x|^{4}-\frac{|x|^{6}-2|x|^{4}+|x|^{2}}{n}\right)_{|x|=1}=0 \\
{\frac{\partial^{2} v}{\partial v_{|x|=1}^{2}}}^{2}=\Lambda^{2} v_{|x|=1}= \\
6\left(2 x_{j}^{2}-8 x_{j}^{2}|x|^{2}+6 x_{j}^{2}|x|^{4}-\frac{6|x|^{6}-8|x|^{4}+2|x|^{2}}{n}\right)_{|x|=1}=0
\end{gathered}
$$

Thus the polynomial constructed in (25) is the solution of the considered Dirichlet problem.

Now consider another Dirichlet problem for the 3 -harmonic equation in the unit ball $\Omega$

$$
\begin{gathered}
\Delta^{3} u(x)=0, \quad x \in \Omega ; \\
u_{\mid \partial \Omega}=0, \quad \frac{\partial u}{\partial v}_{\mid \partial \Omega}=R(x), \quad{\frac{\partial^{2} u}{\partial v}}_{\mid \partial \Omega}=S(x)
\end{gathered}
$$

with polynomial boundary values $R(x)$ and $S(x)$ for $n>2$. In addition to the polynomials $R(x)$ and $S(x)$ consider associated polynomials

$$
\begin{gathered}
R_{(0)}(x)=\frac{1-|x|^{2}}{2} R(x)+\frac{\left(1-|x|^{2}\right)^{2}}{8}(2 \Lambda+1) R(x), \\
S_{(0)}(x)=\frac{\left(1-|x|^{2}\right)^{2}}{8} S(x)
\end{gathered}
$$

$$
\begin{gathered}
R_{(1)}^{\alpha, s}(x)=\left(\Delta(2 \Lambda+1)-2 \frac{1-\alpha}{2 s+4} \Delta^{2}\right) R(x), \\
S_{(1)}(x)=\Delta S(x)
\end{gathered}
$$

where $\alpha \in[0,1]$ и $s \in \mathbb{N}_{0} \equiv \mathbb{N} \cup\{0\}$.

Theorem 5. The solution of the problem (27)-(28) can be written in the form

$$
\begin{gathered}
v(x)=-R_{(0)}(x)+S_{(0)}(x)+ \\
+\frac{\left(1-|x|^{2}\right)^{3}}{16} \int_{0}^{1} \sum_{s=0}^{\infty} \frac{\left(1-\alpha|x|^{2}\right)^{s}(1-\alpha)^{s}}{(2 s) ! !(2 s+2) ! !} \\
\times\left(-\Delta^{s} R_{(1)}^{\alpha, s}+\Delta^{s} S_{(1)}\right)(\alpha x) \alpha^{n / 2-1} d \alpha
\end{gathered}
$$

Proof. Let a biharmonic polynomial $u_{1}(x)$ satisfies the conditions

$$
u_{1}(x)_{\mid \partial \Omega}=R(x)_{\mid \partial \Omega}, \quad \frac{\partial u_{1}}{\partial v}{ }_{\mid \partial \Omega}=\frac{1}{2}(S(x)-R(x))_{\mid \partial \Omega} .
$$

Then the following polynomial

$$
v(x)=\frac{1}{2}\left(|x|^{2}-1\right) u_{1}(x)
$$

is a 3-harmonic polynomial satisfying the conditions $v_{\mid \partial \Omega}=0$ and

$$
\begin{aligned}
\frac{\partial v}{\partial v}_{\mid \partial \Omega}=\Lambda v_{\mid \partial \Omega} & =\left(|x|^{2} u_{1}(x)+\frac{|x|^{2}-1}{2} \Lambda u_{1}(x)\right)_{\mid \partial \Omega} \\
& =u_{1}(x)_{\mid \partial \Omega}=R(x)_{\mid \partial \Omega},
\end{aligned}
$$




$$
\begin{gathered}
{\frac{\partial^{2} v}{\partial v_{\mid \partial \Omega}^{2}}=\left(\Lambda^{2}-\Lambda\right) v_{\mid \partial \Omega}}_{=\left(|x|^{2} u_{1}(x)+2|x|^{2} \Lambda u_{1}(x)\right)_{\mid \partial \Omega}} \\
=\left(R(x)+2 \frac{\partial u_{1}}{\partial v}\right)_{\mid \partial \Omega}=S(x)_{\mid \partial \Omega}
\end{gathered}
$$

The polynomial $u_{1}(x)$ can be written in the form

$$
\begin{aligned}
& u_{1}(x)=R(x)-\frac{|x|^{2}-1}{4}(2 \Lambda R(x)+R(x)-S(x)) \\
& +\frac{\left(|x|^{2}-1\right)^{2}}{8} \int_{0}^{1} \sum_{s=0}^{\infty} \frac{\left(1-\alpha|x|^{2}\right)^{s}(1-\alpha)^{s}}{(2 s) ! !(2 s+2) ! !} \Delta^{s+1} \\
& \times\left(2 \Lambda R+R-S-2 \frac{1-\alpha}{2 s+4} \Delta R\right)(\alpha x) \alpha^{n / 2-1} d \alpha
\end{aligned}
$$

and therefore

$$
\begin{gathered}
v(x)=\frac{|x|^{2}-1}{2} R(x)-\frac{\left(|x|^{2}-1\right)^{2}}{8}((2 \Lambda+1) R(x) \\
-S(x))+\frac{\left(|x|^{2}-1\right)^{3}}{16} \int_{0}^{1} \sum_{s=0}^{\infty} \frac{\left(1-\alpha|x|^{2}\right)^{s}(1-\alpha)^{s}}{(2 s) ! !(2 s+2) ! !} \Delta^{s} \\
\times\left(\Delta(2 \Lambda+1) R-\Delta S-2 \frac{1-\alpha}{2 s+4} \Delta^{2} R\right)(\alpha x) \alpha^{n / 2-1} d \alpha .
\end{gathered}
$$

Taking into account definitions (29) and (30) we obtain formula (31).

Example 4. Let $R(x)=x_{k}^{2}$ and $S(x)=x_{m}$ in the problem (27)-(28). Then

$$
\begin{gathered}
R_{(0)}(x)=\frac{1-|x|^{2}}{2} x_{k}^{2}+5 \frac{\left(1-|x|^{2}\right)^{2}}{8} x_{k}^{2}, \\
S_{(0)}(x)=\frac{\left(1-|x|^{2}\right)^{2}}{8} x_{m} ; \quad R_{(1)}^{\alpha, s}(x)=10, S_{(1)}(x)=0
\end{gathered}
$$

The sum in (31) has the only term indexed by $s=0$. Then we write

$$
\begin{gathered}
v(x)=\frac{|x|^{2}-1}{2} x_{k}^{2}+\frac{\left(|x|^{2}-1\right)^{2}}{8}\left(x_{m}-5 x_{k}^{2}\right) \\
+5 \frac{\left(|x|^{2}-1\right)^{3}}{16} \int_{0}^{1} \alpha^{n / 2-1} d \alpha \\
=\frac{|x|^{2}-1}{2} x_{k}^{2}+\frac{\left(|x|^{2}-1\right)^{2}}{8}\left(x_{m}-5 x_{k}^{2}\right)+5 \frac{\left(|x|^{2}-1\right)^{3}}{8 n} .
\end{gathered}
$$

Let us verify that the obtained polynomial $v(x)$ is really the solution of the problem (27)-(28) with $R(x)=x_{k}^{2}$ and
$S(x)=x_{m}$. Using formula (26) from Example 3 we can conclude that the polynomial $v(x)$ is a 3-harmonic polynomial

$$
\Delta^{3} v(x)=-\frac{5}{8} \Delta^{3}\left(x_{k}^{2}|x|^{4}-\frac{|x|^{6}}{n}\right)=0 .
$$

In addition to this, the polynomial $v(x)$ satisfies the conditions (28) with $R(x)=x_{k}^{2}$ and $S(x)=x_{m}$. Indeed

$$
\begin{gathered}
v_{|x|=1}=0 \\
\frac{\partial v}{\partial v_{|x|=1}}=\Lambda v_{|x|=1}= \\
\left(x_{k}^{2}|x|^{2}+\left(|x|^{2}-1\right) x_{k}^{2}+\frac{\left(|x|^{2}-1\right)|x|^{2}}{2}\left(x_{m}-5 x_{k}^{2}\right)+\right. \\
\left.+\frac{\left(|x|^{2}-1\right)^{2}}{8}\left(x_{m}-10 x_{k}^{2}\right)+15 \frac{\left(|x|^{2}-1\right)^{2}|x|^{2}}{4 n}\right)_{|x|=1}=\left(x_{k}^{2}\right)_{|x|=1},
\end{gathered}
$$

and by Lemma 4 we find

$$
\begin{gathered}
{\frac{\partial^{2} v}{\partial v_{|x|=1}^{2}}}=\left(\Lambda^{2}-\Lambda\right) v_{|x|=1}= \\
\left(4 x_{k}^{2}|x|^{2}+2|x|^{2} x_{k}^{2}+|x|^{4}\left(x_{m}-5 x_{k}^{2}\right)\right)_{|x|=1}-\left(x_{k}^{2}\right)_{|x|=1} \\
=\left(x_{m}\right)_{|x|=1} .
\end{gathered}
$$

Therefore the polynomial $v(x)$ is the solution of the considered Dirichlet problem.

On the base of Theorems 3, 4 and 5 we can get the following general statement.

Theorem 6. The solution of the Dirichlet problem

$$
\begin{gathered}
\Delta^{3} u(x)=Q(x), \quad x \in \Omega \\
u_{\mid \partial \Omega}=P(x), \quad \frac{\partial u}{\partial v}_{\mid \partial \Omega}=R(x),{\frac{\partial^{2} u}{\partial v^{2}}}_{\mid \partial \Omega}=S(x)
\end{gathered}
$$

in the unit ball $\Omega$ with the polynomial data $Q(x), P(x)$, $R(x)$ and $S(x)$ has the form

$$
\begin{gathered}
u(x)=P_{(0)}(x)-R_{(0)}(x)+S_{(0)}(x) \\
+\frac{\left(1-|x|^{2}\right)^{3}}{16} \int_{0}^{1} \sum_{s=0}^{\infty} \frac{\left(1-\alpha|x|^{2}\right)^{s}(1-\alpha)^{s}}{(2 s) ! !(2 s+2) ! !} \Delta^{s} \\
\times\left(P_{(1)}^{\alpha, s}-R_{(1)}^{\alpha, s}+S_{(1)}-Q_{(1)}^{\alpha, s}\right)(\alpha x) \alpha^{n / 2-1} d \alpha
\end{gathered}
$$

where polynomials $P_{(0)}(x), R_{(0)}(x)$ and $S_{(0)}(x)$ are defined by (22), (29), polynomial $P_{(1)}^{\alpha, s}(x), R_{(1)}^{\alpha, s}(x)$ and 
$S_{(1)}(x)$ are defined by (23), (30), and polynomial $Q_{(1)}^{\alpha, s}(x)$ has the form

$$
Q_{(1)}^{\alpha, s}(x)=\frac{(1-\alpha)^{2}}{(2 s+4)(2 s+6)} Q(x) .
$$

Proof. It is not hard to see that the solution of the problem (32)-(33) can be decomposed into the sum of solutions of three problems: (3)-(4), (20)-(21) and (27)-(28). The sum of these solutions obtained by formulas (18), (24) and (31), correspondingly, give us the desired solution (34).

Example 5. With the help of the software package "Mathematica" which make symbol calculations we find by formula (34) the solution of the following Dirichlet problem

$$
\begin{aligned}
& \Delta^{3} u(x)=x_{1}^{2}-2 x_{3}^{2}, \quad x \in \Omega \subset \mathbb{R}^{3} ; \\
& u_{\partial \Omega \Omega}=\left(x_{1}^{4} x_{2}^{2}-x_{2} x_{3}^{5}\right)_{\mid \partial \Omega},\left.\frac{\partial u}{\partial v}\right|_{\partial \Omega}=\left(x_{1}^{6}+2 x_{2}^{4} x_{3}^{2}\right)_{\mid \partial \Omega} \text {, } \\
& \frac{\partial^{2} u}{\partial v^{2}}=\left(x_{2} x_{3}^{5}-3 x_{1}^{2} x_{2}^{4}\right)_{\mid \partial \Omega}
\end{aligned}
$$

Defining for short $|x|^{2}=x_{1}^{2}+x_{2}^{2}+x_{3}^{2}$ and omitting intermediate calculations we have

$$
\begin{gathered}
u\left(x_{1}, x_{2}, x_{3}\right)=x_{1}^{4} x_{2}^{2}-x_{2} x_{3}^{5} \\
+\frac{|x|^{2}-1}{2}\left(x_{1}^{6}-6 x_{1}^{4} x_{2}^{2}+2 x_{2}^{4} x_{3}^{2}+6 x_{2} x_{3}^{5}\right) \\
-\frac{\left(|x|^{2}-1\right)^{2}}{8}\left(13 x_{1}^{6}-48 x_{1}^{4} x_{2}^{2}+3 x_{1}^{2} x_{2}^{4}\right. \\
\left.+26 x_{2}^{4} x_{3}^{2}+47 x_{2} x_{3}^{5}\right) \\
+\frac{\left(|x|^{2}-1\right)^{3}}{665280}\left(71269+870840 x_{1}^{4}+210600 x_{2}^{4}\right. \\
-592200 x_{2}^{3} x_{3}+4715 x_{3}^{2}-48960 x_{3}^{4} \\
+1800 x_{2} x_{3}\left(253+1645 x_{3}^{2}\right)-5 x_{1}^{2} \\
\left(-57979+458064 x_{2}^{2}+118440 x_{2} x_{3}+57888 x_{3}^{2}\right) \\
\left.+15 x_{2}^{2}\left(317+74784 x_{3}^{2}\right)\right) .
\end{gathered}
$$

\section{Acknowledgements}

This paper is financial supported by the Ministry of Education of Russian Federation, grant No. 14.B37.21.0613.

\section{References}

[1] E. Almansi. Sull'integrazione dell'equazione differenziale $\Delta^{2 n} u=0$. Ann. Mat. Pura Appl., (3) 2 1899, pp.1-51.

[2] V. Karachik. On an expansion of Almansi type. Mathematical Notes, 83:3-4, 2008, pp. 335-344.

[3] N. Nicolescu. Probléme de lánalyticité par rapport á un opérateur linéaire. Studia Math., 16, 1958, pp. 353-363.

[4] V. Karachik. Application of the Almansi formula for constructing polynomial solutions to the Dirichlet problem for a second-order equation. Russian Mathematics, vol. 56, Issue 6, June 2012, pp. 20-29.

[5] V.S. Vladimirov i dr. Sbornik zadach po uravneniyam matematicheskoi fiziki, Fizmatlit, 2001 (in Russian).

[6] V. Karachik. On one set of orthogonal harmonic polynomials. Proceedings of American Mathematical Society, 126:12, 1998, pp. 3513-3519.

[7] H. Bateman and A. Erdélyi. Higher Transcendental Functions, vol. 2, New York, 1953.

[8] V. Karachik, N. Antropova. On the solution of the inhomogeneous polyharmonic equation and the inhomogeneous Helmholtz equation. Differential Equations, $46: 3,2010$, pp. 387-399.

[9] V. Karachik. Construction of polynomial solutions to some boundary value problems for Poisson's equation. Computational Mathematics and Mathematical Physics, 51:9, 2011, pp. 1567-1587.

[10] V. Karachik, N. Antropova. Construction of polynomial solutions to the Dirichlet problem for the biharmonic equations in a ball. Vestnik SUSU, seriya "Mathematika. Mechanika. Phisika", 32(249):5, 2011, pp. 39-50 (in Russian).

[11] V. Karachik. On one representation of analytic functions by harmonic functions. Siberian Advances in Mathematics, 18:2, 2008, pp. 142-162.

[12] D. Khavinson, H.S. Shapiro. Dirichlet's problem when the data is an entire function. Bull. London Math. Soc., 24, 1992, pp. 456-468.

[13] H. Render. Real Bargmann spaces, Fischer decompositions and sets of uniqueness for polyharmonic functions. Duke Math. J., 142, 2008, pp. 313-352.

[14] V. Karachik. A problem for the polyharmonic equation in the sphere. Siberian Mathematical Journal, 32:5, 2005, pp. 767-774.

[15] V. Karachik. Method for constructing solutions of linear ordinary differential equations with constant coefficients. Computational Mathematics and Mathematical Physics, 52:2, 2012, pp. 219-234.

[16] V. Karachik, B.Kh. Turmetov, A. Bekaeva. Solvability conditions of the Neumann boundary value problem for the biharmonic equation in the unit ball. International Journal of Pure and Applied Mathematics, vol. 81, No. 3, 2012, 487-495.. 